\title{
The Quantized Characterization of Cooked Rice Hardness and Research on the Automatic Measurement Technology
}

\author{
Nan Jiang ${ }^{1}$, Yuan $\mathrm{Gao}^{2}$, Jiping Zhou ${ }^{1}$, Longqin Gao ${ }^{1}$, Jianhua Zhou ${ }^{1}$, Qigen Dai ${ }^{2}$ \\ ${ }^{1}$ Mechanical Engineering College, Yangzhou University, Yangzhou, China \\ ${ }^{2}$ Agricultural College, Yangzhou University, Yangzhou, Jiangsu, China \\ E-mail:jpzhou@yzu.edu.cn \\ Received August 27, 2009; revised September 19, 2009; accepted October 4, 2009
}

\begin{abstract}
The hardness of cooked rice is one of the most important criteria which determine the rice quality. The commonly used near-infrared reflectance (NIR) method is still in argument due to its indirectness and possible error. In this paper, a mechanical method was proposed and its principle, automation, components and operative reliability were evaluated and compared with the NIR method. The results showed that the mechanical testing method can accurately detect the rice quality and were consistent with the NIR testing data. This new mechanical method can be effectively used in rice quality testing and branding with the advantage of simplicity, accuracy and reliability.
\end{abstract}

Keywords: Rice Hardness, Measurement System, Stress-Strain

\section{Introduction}

Rice is one of the most important food crops in China, and China also is the biggest country of production and consumption of rice. The people's requirement of high quality rice is increased with time [1]. The requirement of high quality rice will be substantially increasing in market demand. Therefore, the study and identification of rice eating quality will be more emphasized.

The rice hardness is one of the important factors affecting rice eating quality. The existing detection methods of rice hardness are sensory evaluation [2] and physical-chemical analysis [3]. The former has established a series of the evaluation standards, and these have some guiding effects to the rice quality testing. But it also has some uncertain factors and thus is lack of public reliability and persuasion. Researchers are searching a conformable and scientific method to test the quality of rice and other organic solids. Procter (1955) have putted forward the standard of chew food, and use the human tactility to research the physical characteristics of food [4]. Procter and Szczeniak (1963) have confirmed the Texture Profile Analysis to describe the quality of food, and use the stress-time curve to show the food quality [5]. Recent years researchers use the Texture Analyzer and Instron Universal Texting Machine to test the cooked-rice quality especially the hardness and viscosity, but the method's adaptability is few to testify. Meanwhile the Taste Ana- lyzer has been developed to test the rice quality [6], which is based on the NIR (Near-Infrared Reflectance) technique, analyzes the rice quality components (protein, starch, oil, sugar and water etc), and finally indirectly get the appearance, hardness, viscosity, equilibrium degree and taste data of rice., This method obtains the result from conversion of a rage of experiential formulas, not through the press the rice directly to get the testing result, so its analysis result is not very precise. Besides, another technique is use the Image Reconstruction based on Analytic Method to test the brightness and glossiness of rice, to be brief is through the appearance to judge the eating quality of cooked rice [7].

Therefore, the establishment of a new method and technique to reflect accurately the rice hardness and other characteristic parameters is very important for rice grade identification and quality breeding through modern genetic engineering.

\section{The Measurement and Analysis of Rice Hardness}

\subsection{The Rice Hardness and Stress Analysis}

The rice hardness means the resistance of the cooked rice to external-introduced pressure. When people chew the rice, they can intuitively feel its hardness or softness. When the external pressure reaches a critical value, the 
rice will break thoroughly, and this critical value that can be used to characterize the rice hardness is usually between $0 \sim 150 \mathrm{~g}$ force and the strain is between $0 \sim 3 \mathrm{~mm}$, so the key point is to get the stress-strain relationship of the rice.

\subsection{The Basic Ideas of Measurement}

Now there is still not a mature and reliable method and technique for micro-stress and displacement measurement. Based on analysis of existing methods and technology of food testing [8-10], correct understanding and grasp of rice relationship between the material properties, machinery mechanics measurement techniques were used to establish a measurement of rice stress-strain, according to the characteristics of small size, displacement and force. The method developed in this study could measure the rice force which could be conversed to express the rice hardness.

\subsection{The Basic Principles of Measurement}

The basic principles of measurement are the double cantilevers stress and strain measurement techniques as shown in Figure 1(a). The rice to be measured is put on the pallet between the double cantilevers, external force push the indenter, which fixed on the up-cantilever, to oppress the rice, then the rice will press the down-cantilever. There are 8 resistance strain gauges (BHF3503AA type) in Figure 1(b), and the Figure 1(c) shows that the strain gauges $1 \sim 4$ are linked with differential circuit to detect the double cantilevers relative deformation that is the strain of rice; meanwhile, the strain gauges $5 \sim 8$ are linked with another differential circuit to detect the force of down-cantilever that is the stress of the tested rice. Then through the data wire transfer the stress-strain signal of rice, which acquired by the data acquisition board, then analysis the data to obtain the rice hardness finally.

\section{The Measurement System}

Figure 2 shows the basic ideas and principles for the

measurement of the rice, and the automatic system used to measure the rice hardness.

\subsection{The Machinery of Automatic Force Application}

The rice automatic measurement system is composed by motor, transmission shaft, cam, guide rod and spring etc. as shown in Figure 3. The principle is that when the motion control system sends a signal to the motor, the motor drives the cam to rotate through the transmission shaft, the cam presses the up-cantilever through the guide rod, meanwhile press the rice. The loading stroke decided by the size of the rice (usually is the $85 \%$ of the rice), and the maximum loading stroke is the net volume of the cam. The control program detects the deformation of the rice to judge whether it reaches the loading stroke. When the deformation reaches the stroke, the motor will stop loading and drive the cam to reverses to the initial position.

\subsection{Measurement and Date Acquisition System}

In order to get the measured value of micro-stress and strain of different materials, the double cantilevers' high

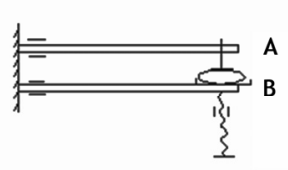

a)

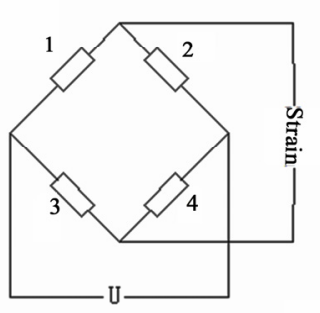

c)

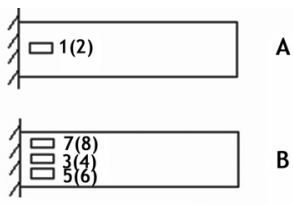

b)

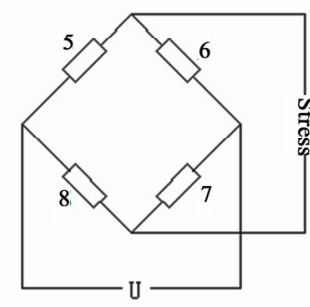

Figure 1. Schematic diagram of measurement principle.

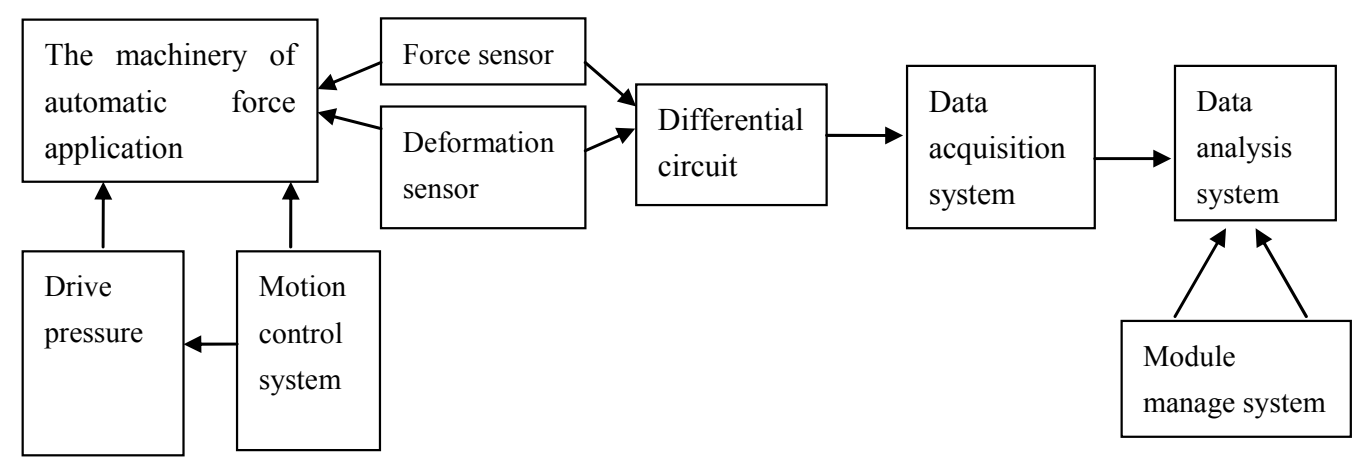

Figure 2. The components of automatic measurement system. 
ratio of length and width is 200:15:1 determined preliminarily. Besides, we choose the AAA resistance strain gauge, and the requirements of patch, substrate and cure are very strictly designed, and measurement accuracy is ensured by means of the differential circuit and compensation (Figure 1(c)). The deformation signals of the double cantilevers are collected by the special data acquisition board, and then through the data wire linked to the computer.

\subsection{Data Analysis System}

Language Delphi 7 is used to compile the program of data analysis system, draw the "stress-strain", "stress-time" and "strain-time" curves, and to calculate the maximum stress and eigenvalue, record and save the data of the rice (Figure 4).

\section{The System Operation and Test Verification}

\subsection{The Initialization of the Measurement System}

Figure 5 shows that the automatic measurement system designed and manufactured by ourselves, composed by automatic measurement equipment, data wire and computer.

\subsubsection{Stress-Strain Parameter Calibration}

In order to get the relationship between stress-strain, micro-force and deformation, and to ensure the accuracy before the first time to run the system, we demarcate both force signal and strain signal. The force signal of the up-cantilever is demarcated by the spiral micrometer, it presses the up-cantilever $0.2 \mathrm{~mm}$ each time, and the strain signal of the down-cantilever is demarcated by weights, they press the down-cantilever $10 \mathrm{~g}$ each time. The demarcation value of force and strain signal is listed in Table 1 and Table 2. The average of the three demarcation values is used as the basis. According to the Table 1 and Table 2 the linearity of the demarcation values is good, the data acquisition system can meet the requirement.

\subsubsection{System Self-Check}

After demarcating, the system must self-check. According to the theory, without the test object, the double cantilevers are rigid connection, when the machinery of automatic force application press the double cantilevers the relative deformation should be zero, and the stressstrain curve is straight line of which the angle is $45^{\circ}$ with the coordinate axis, meanwhile the curves of loading and unloading should be coincident in Figure 6 which shows that the system is accurate and reliable.

\subsection{The Preparation of Test}

\subsubsection{Testing Scheme}

According to the evaluation standard of the rice quality, in order to verify the adaptability, reliability and accuracy of the rice hardness test system, we establish the testing scheme as follows:

Firstly, aiming at the same variety rice using the different types of indenters to test the rice, and analysis the

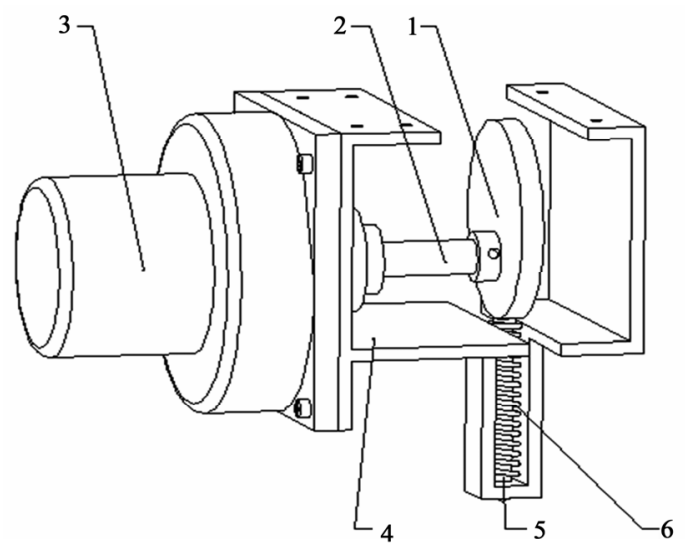

1.Cam 2.Transmission shaft 3.Motor 4.Box 5.Guide cod 6.Spring

Figure 3. The machinery of automatic force application.

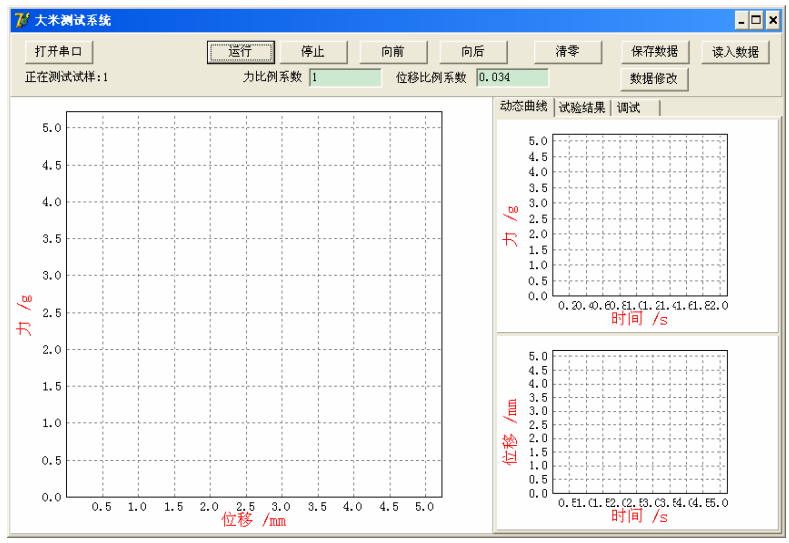

Figure 4. The interface of data analysis system.

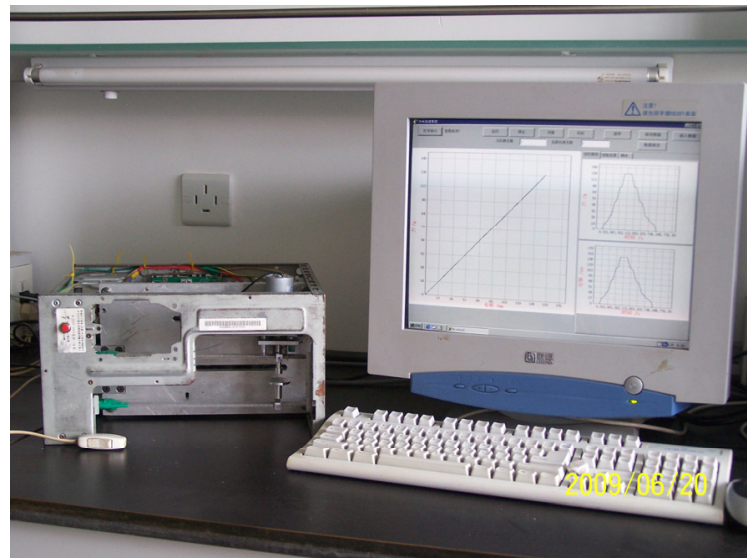

Figure 5. Automatic measurement system. 
Table 1. Force demarcation.

\begin{tabular}{cc|cc}
\hline $\begin{array}{c}\text { Standard } \\
\text { force } \\
(\mathrm{g})\end{array}$ & $\begin{array}{l}\text { Force } \\
\text { signal } \\
\text { equivalent }\end{array}$ & $\begin{array}{c}\text { Standard } \\
\text { force } \\
(\mathrm{g})\end{array}$ & $\begin{array}{l}\text { Force } \\
\text { signal } \\
\text { equivalent }\end{array}$ \\
\hline 0 & 0.0 & 90 & 1033.3 \\
10 & 86.0 & 100 & 1166.7 \\
20 & 255.0 & 110 & 1213.3 \\
30 & 335.3 & 120 & 1350.0 \\
40 & 465.7 & 130 & 1500.0 \\
50 & 563.3 & 140 & 1636.7 \\
60 & 686.7 & 150 & 1716.7 \\
70 & 813.3 & 160 & 1843.3 \\
80 & 920.0 & & \\
\hline
\end{tabular}

Table 2. Strain demarcation.

\begin{tabular}{cc|cc}
\hline $\begin{array}{c}\text { Standard } \\
\text { strain } \\
(\mathrm{mm})\end{array}$ & $\begin{array}{c}\text { Strain signal } \\
\text { equivalent }\end{array}$ & $\begin{array}{c}\text { Standard } \\
\text { strain } \\
(\mathrm{mm})\end{array}$ & $\begin{array}{c}\text { Strain signal } \\
\text { equivalent }\end{array}$ \\
\hline 0 & 0.0 & 1.6 & 175.3 \\
0.2 & 22.5 & 1.8 & 197.0 \\
0.4 & 42.7 & 2.0 & 219.0 \\
0.6 & 65.8 & 2.2 & 241.0 \\
0.8 & 86.0 & 2.4 & 262.0 \\
1.0 & 112.7 & 2.6 & 280.0 \\
1.2 & 131.7 & 2.8 & 301.3 \\
1.4 & 153.7 & 3.0 & 322.0 \\
\hline
\end{tabular}

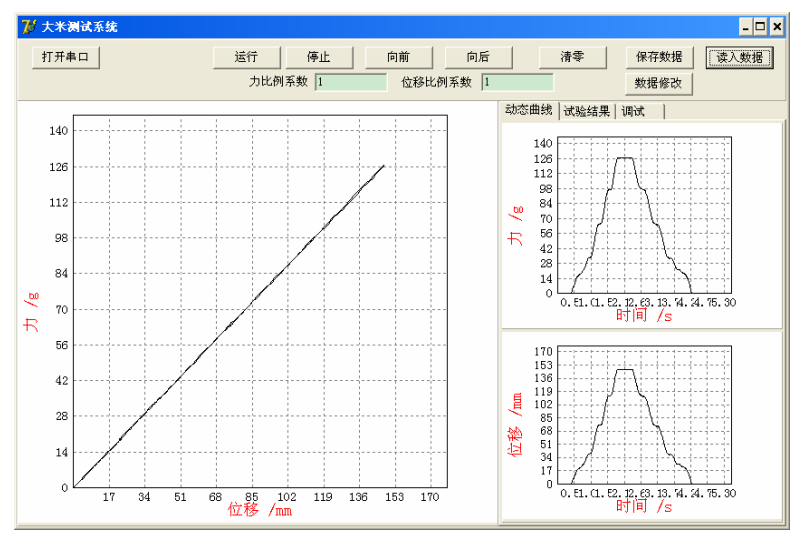

Figure 6. The graph of system self-check.

consistency of the rice under the same indenter;

Secondly, aiming at the varied rice varieties, using the same type of the indenter to test the stress-strain, and verifying if the test system can discriminate the difference of varied rice varieties;

Thirdly, using one of the indenters to test the same variety at different time, and verifying if the test system could discriminate the change of the results with different time.

We select the five rice varieties randomly, and take the average of the test results as the measured value of the rice.

\subsubsection{Preparation of Need Checking Sample}

1) Preparation of the test rice

According to the standard cooking method [11] to pre- pare the test rice: 1) use the electronic balance to weigh $30 \mathrm{~g}$ rice; 2) wash the rice four times, and add the 1.33-times water; 3 ) immersing the rice 30 minutes, then cooking 30 minutes; 4 ) after the rice cooked standing it 10 minutes, then cooling it 20 minutes in the cooling equipment; 5) extract the sample to test during which the sample is stirred to maintain uniformly.

2) Preparation of the indenters

For the requirement of the test, at the beginning of the scheme establishment we use the different indenters to test the rice hardness, in order to get the suitable one. We prepare the $\Phi 2 \mathrm{~mm}$ and $\Phi 3 \mathrm{~mm}$ spherical indenter, $\Phi 2 \mathrm{~mm}$ and $\Phi 3 \mathrm{~mm}$ plane indenter, $\Phi 10 \mathrm{~mm}$ and $\Phi 15 \mathrm{~mm}$ cylindrical indenter, $10 \mathrm{~mm} \times 1 \mathrm{~mm}$ tool form indenter, respectively, while the cylindrical indenter is used to test the rice-flour dough mainly.

\subsection{Measurement Results and Analysis}

1) Choose the first class rice (according to National Standard), and use the different indenters to test it, the measured results are showed in Table 3 . We can educe that the stresses are different with the different indenters, and the average stresses exhibit a obvious difference. But the slope of the stress-strain curves has a good consistency. Therefore, we could use the slope to express the hardness of the cooked rice, and the big slope indicates a high hardness.

Note: the average is the ratio of maximum force to area of the indenter

2) Table 4 shows data of using the $\Phi 3 \mathrm{~mm}$ plane indenter to test the first class rice and the data of Baoying lake organic rice at the different time is also listed in Table 4 . The all listed results show that the testing system can distinguish the difference of the hardness of the rice at the different time.

3) Table 5 shows data of using the $\Phi 10 \mathrm{~mm}$ cylindrical indenter to test the six different breed rices. We can see that the testing system could distinguish the variance of the rice hardness.

4) Comparison with the Japanese taste analyzer

According to the test results of hardness which is tested through the Taste Analyzer STA 1A indirectly, we use the rice under the same cooking standard to test, the results are in Table 5. The hardness of the same variety exhibits the consistency between the automatic testing system and the Japanese taste analyzer, which prove the correctness of the system.

5) Analysis of system measuring error

According to the test scheme, every variety repeats 5 times. Take the first class rice as example, use the $\Phi 3 \mathrm{~mm}$ plane indenter to test, under the cooking standard the test values are in Table 7. The absolute error is 0.2691 , and the average of the relative error is $1.35 \%$, which shows that the error and the reproducibility of the measuring system can meet the test requirement. 
Table 3. The data of the same breed rice with different pressure head.

\begin{tabular}{cccc}
\hline Indenter & The maximum force $(\mathrm{g})$ & Average $\left(\mathrm{g} / \mathrm{mm}^{2}\right)$ & The slope of the curve \\
\hline Ф3 plane indenter & 41.2224 & 5.4990 & 19.9910 \\
$\Phi 3$ spherical indenter & 39.7255 & 5.3949 & 19.4340 \\
$\Phi 2$ plane indenter & 38.6891 & 13.1215 & 19.8397 \\
$\Phi 2$ spherical indenter & 37.3744 & 12.7033 & 19.7861 \\
Tool form on lateral & 37.4714 & 17.0325 & 19.8395 \\
Tool form on vertical & 38.1325 & 4.4340 & 19.4976 \\
\hline
\end{tabular}

Table 4. The data of the same rice variety at different time.

\begin{tabular}{ccc}
\hline Time & First class according rice & Baoying lake organic rice \\
\hline 0 hour & 19.7433 & 20.7861 \\
1 hour & 19.7503 & 21.1354 \\
2 hour & 19.9474 & 21.4977 \\
6 hour & 20.3053 & 21.8576 \\
\hline
\end{tabular}

Table 5. The data of contrast experiment.

\begin{tabular}{ccc}
\hline \multirow{2}{*}{ Rice breed } & Auto-test system & Taste analyzer \\
\cline { 2 - 3 } & The slope & Hardness \\
\hline Daoya rice & 21.5191 & 8.1 \\
Baoying lake organic rice & 21.0686 & 7.9 \\
San'an rice & 20.9645 & 7.6 \\
Yueguang rice & 20.8404 & 6.8 \\
Xinxie rice & 20.3684 & 6.5 \\
The first class rice & 20.3248 & 6.3 \\
\hline
\end{tabular}

Table 7. The data of error analysis.

\begin{tabular}{ccccccc}
\hline No. & 1 & 2 & 3 & 4 & 5 & Average \\
\hline Test values & 19.7433 & 19.9509 & 19.9876 & 20.0124 & 19.9473 & 19.9283 \\
Absolute error & 0.2691 & Relative error & & $1.35 \%$ \\
\hline
\end{tabular}

\section{Conclusions}

A mechanical automatic testing system is established to quantitatively characterize the rice quality such as its hardness. Through test the deformation of the rice, we can use the slope of the curve to express the rice hardness as the evaluation standard of the rice. The test results show that the automatic testing system could detect the hardness difference of varied rice varieties, and it also could detect the change of one variety hardness with time, the testing errors and reproducibility are in accordance with the demand of the rice identification. The test results are in consistent with that of the Taste Analyzer.

This automatic testing method based on mechanical theory is feasible, it can provides a convenient, accurate and reliable method to quantitatively obtain the rice parameters, and is important for studying and characteriz- ing the quality of organic solid substance. Further improvements are still needed in testing precision, reducing testing error, and using in other parameter measurement of rice.

\section{Acknowledgement}

This research is supported by the National Technology R\&D Program of China (Contract No. 2006BAD02A03)

\section{References}

[1] J. D. Jin and S. Y. Zhang, "Quality traits of high-quality rice at home and abroad research (in Chinese)," Chinese Journal of Jilin Agricultural Sciences, Vol. 28, No. 6, pp.13-15, 2003

[2] W. Y. Fu and Y. W. Xing, "Grain quality evaluation of 
current situation and prospect (in Chinese)," Chinese Journal of Natural Science Journal of Hainan University, Vol. 14, No. 3, pp. 269 271, September 1996.

[3] X. M. Zhang, Y. C. Wang etc, "Rice taste evaluation of the progress (in Chinese)," Chinese Journal of Seed, Vol. 1, pp. 52-53, 2002.

[4] B. E. Procter, "Instrement evaluation and initial test," Chinese Journal of Food Technology, Vol. 9, pp. 417441, 1955.

[5] A. S. "Szczeniak objective measurements of food texture," Chinese Journal of Food Science, Vol. 28, pp. 420-441, 1963.

[6] X. M. Zhan, T. S. Zheng, and J. H. Tao, "Study on application of texture analyzer in quality evaluation of ric (in Chinese)," Chinese Journal of Food Science, Vol. 28, No. 9, pp. 62-65. 2007.

[7] A. H. Cheng, X. H. Li, X. M. Yao etc, "Study on the correlation between image analysis properties and rice sensory evaluation of japonica rice in northeast china (in Chinese)," Chinese Journal of Grain and Oil Food, Vol. 14, No. 4, pp. 25-27, 2006.

[8] X. F. Guo and Y. D. Mu, "Evaluation of a method for determining texture characteristics of cooked rice (in Chinese)," Chinese Journal, Journal of the Chinese cereals and oils association, Vol. 4, No. 21, pp. 9-11, 2006.

[9] Y. Lan, O. R. Kunze, "Fissure resistance of rice varieties," Chinese Journal Applied Engineering in Agriculture, Vol. 12, No. 3, pp. 365-368, 1996.

[10] Y. Lan and O. R. Kunze, "Fissure characteristics related to moisture adsorption stresses in rice," Chinese Journal Transactions of the ASAE, Vol. 39, No. 6, pp. 21682174, 1996.

[11] "People's Republic of China national standard quality assessment tests of rice cooking," GB/T 15682, 1995. 